\title{
Intraoperative Real-Time Visualization of the Lymphatic Vessels Using Microscope-Integrated Laser Tomography
}

\author{
Akitatsu Hayashi, MD ${ }^{1, *}$ Hidehiko Yoshimatsu, MD ${ }^{2, *}$ Giuseppe Visconti, MD, $\mathrm{PhD}^{30}$ \\ Sasithorn Sujarittanakarn, $\mathrm{MD}^{4}$ Guido Giacalone, MD, $\mathrm{PhD}^{5}$ Nobuko Hayashi, $\mathrm{MD}^{6}$ \\ Takumi Yamamoto, MD, $\mathrm{PhD}^{7}$ Johnson Chia-Shen Yang, MD, FACS ${ }^{8}$ Joon Pio Hong, MD, PhD, MMM ${ }^{90}$
}

${ }^{1}$ Department of Lymphedema Center, Kameda General Hospital, Chiba, Japan

2 Department of Plastic Surgery, Cancer Institute Hospital of the JFCR, Tokyo, Japan

3 Department of Plastic and Reconstructive Surgery, University Hospital "A. Gemelli," Università Cattolica del "Sacro Cuore," Rome, Italy

${ }^{4}$ Department of Surgery, Faculty of Medicine, Thammasat University, Bangkok, Thailand

${ }^{5}$ Department of Lymphatic Surgery, AZ Sint-Maarten Hospital, Duffel, Belgium

${ }^{6}$ Department of Plastic Surgery, Taiyo-kai Social Welfare Awachiiki Iryo Center, Chiba, Japan
Address for correspondence Akitatsu Hayashi, MD, Department of Lymphedema Center, Kameda General Hospital, 929 Higashi-cho, Kamogawa City, Chiba, Japan 296-8602

(e-mail: promise6me5now@gmail.com).

${ }^{7}$ Department of Plastic and Reconstructive Surgery, National Center for Global Health and Medicine, Tokyo, Japan

8 Division of Plastic and Reconstructive Surgery, Department of Surgery, Kaohsiung Chang Gung Memorial Hospital, Chang Gung University College of Medicine, Kaohsiung, Taiwan

${ }^{9}$ Department of Plastic and Reconstructive Surgery, Asan Medical Center, University of Ulsan College of Medicine, Seoul, Korea

J Reconstr Microsurg 2021;37:427-435.

\section{Abstract}

Keywords

- lymphedema

- lymphatic

- imaging

- LVA

- laser tomography
Background Detection and selection of the lymphatic vessels are important for maximizing therapeutic efficacy of lymphaticovenular anastomosis (LVA). Some imaging modalities have been reported to be useful for intraoperative identification of the lymphatic vessels, but they have limitations. In this article, we present new capabilities of intraoperative laser tomography, which was used to evaluate the lumen of the lymphatic vessel and to validate the patency of anastomosis.

Methods Fifty-two patients with upper extremity lymphedema secondary to breast cancer treatment underwent indocyanine green (ICG) lymphography and real-time laser tomography imaging of ICG-enhanced lymphatic vessels intraoperatively before transecting the vessels during LVA. The imaging findings of the lymphatic vessels in laser tomography were investigated. Time required for scanning of the lymphatic vessels was compared between laser tomography and ultrasonography. The correlation between the thickness of the lymphatic vessel wall measured with laser tomographic imaging and the histologically measured thickness of the lymphatic vessel wall was examined. The patency of anastomosis sites was determined based on the image using laser tomography immediately after establishment of LVA.

Results A total of 132 ICG-enhanced lymphatic vessels were scanned with laser tomography showing clear lumen with surrounding vessel wall. The required time for lymphatic vessel scanning was significantly shorter with laser tomography than with

These authors contributed equally to this work.

received

May 24, 2020

accepted

September 2, 2020

published online

October 14, 2020 (c) 2020. Thieme. All rights reserved.

Thieme Medical Publishers, Inc.,

333 Seventh Avenue, 18th Floor,

New York, NY 10001, USA
DOI https://doi.org/

10.1055/s-0040-1718549.

ISSN 0743-684X. 
ultrasonography ( $1.6 \pm 0.3$ vs. $4.8 \pm 1.2$ minutes; $p=0.016)$. Strong correlation was seen between the thickness of the lymphatic vessels wall measured using laser tomography and the histologically measured thickness of the lymphatic vessel wall $(r=0.977,95 \%$ confidence interval: $0.897-0.992, p<0.001)$. The quality of patency was evaluated immediately after anastomosis, which assisted in deciding whether reanastomosis was needed.

Conclusion Microscope-integrated laser tomography provides real-time images of the lymphatic vessels in extremely high resolution and enables evaluation of lymphatic lumen condition and objective post-LVA anastomosis status.

Lymphaticovenular anastomosis (LVA) is effective and minimally invasive treatment for refractory lymphedema. ${ }^{1-3}$ The lymphatic vessels become sclerotic over time after lymph flow obstruction and subsequently lose their function to drain lymph fluid in peripheral lymphedema patient. ${ }^{2}$ "Functional" lymphatic vessels are those retaining its ability to transport lymph. Establishing bypass to nonfunctional, or severely sclerosed, lymphatic vessels leads to minimal therapeutic effect in LVA. Therefore, identification of functional lymphatic vessels is imperative for effective LVA. ${ }^{3,4}$ Microscope-integrated indocyanine green (ICG) fluorescence imaging or other ICG fluorescence imaging tools are often used for this purpose intraoperatively. Although ICG-enhanced lymphatic vessels had been considered "functional" for its capability to transport contrast-containing lymph and nonICG-enhanced lymphatic vessels "nonfunctional," it was reported that there are some lymphatic flow-positive, and thus functional, lymphatic vessels that are not enhanced by ICG fluorescence imaging. ${ }^{5}$ Recently, ultrasound has become a valuable and powerful tool for microsurgery and supermicrosurgery. ${ }^{6}$ Hayashi et al reported noninvasive ultrasound visualization of the lymphatic vessels to be useful for selecting appropriate lymphatic vessels for LVA, not only preoperatively but also intraoperatively, especially in regions masked by dermal backflow pattern of ICG lymphography. ${ }^{3,4,7}$ However, disadvantages of ultrasound systems are that it is highly operator dependent and time consuming. In addition, assessment of the degenerative status of the lymphatic vessels is still not possible with any modality. If the thickness of the lymphatic vessel wall and the diameter of the lumen can be measured before transecting a lymphatic vessel, higher efficacy of LVA can be expected. Furthermore, if the patency of an anastomosis can be confirmed immediately after LVA, even in small vessels, it should also contribute to better results.

In this article, we present new capabilities of laser tomography for imaging of the lymphatic vessels for intraoperative selection of appropriate lymphatic vessels and for evaluation of immediate postanastomosis patency.

Laser tomography has significantly refined clinicians' approach to diagnosis, management, and surveillance of ophthalmic diseases. ${ }^{8,9}$ Laser tomography is an imaging modality that uses reflection of near-infrared light to generate images and provides high-resolution cross-sectional and optical co- herence tomographic data. Using this technology, surgeons would potentially have immediate feedback on completion of surgical tasks or new understanding of the anatomic configuration of the tissues. Laser tomography could provide specific real-time guidance for surgical manipulations and selection of functional lymphatic vessels for LVA. To the best of our knowledge, this is the first study to focus on imaging of the lymphatic vessels using intraoperative real-time microscopeintegrated laser tomography.

\section{Patients and Methods}

This is a single-site multisurgeon investigational device study. Fifty-two female patients with upper extremity lymphedema secondary to breast cancer treatment underwent ICG lymphography and subsequent LVA from December 2018 to April 2019. All patients suffered from progressive lymphedema refractory to conservative treatment and had no history of surgical treatment for lymphedema. This study was conducted under the Institutional Ethical Review Board. All patients provided written informed consent for participation in this retrospective observational study.

ICG was injected to all patients as reported previously on the day before surgery for decision of incision point for LVA and intraoperative identification of the lymphatic vessels: $0.2 \mathrm{~mL}$ of ICG (Diagnogreen 0.25\%; Daiichi Pharmaceutical, Tokyo, Japan) was injected subcutaneously into the affected upper extremities at the second web space of the hand and the ulnar border of the palmaris longus tendon at the level of the wrist. ${ }^{10}$ After ICG injection, circumferential fluorescent images of lymphatic drainage channels were obtained using an infrared camera system preoperatively (Photodynamic Eye; Hamamatsu Photonics K.K., Hamamatsu, Japan) and intraoperatively (LIGHTVISION; Shimadzu Corporation, Kyoto, Japan). LVA was performed at a site distal to the place where a dermal backflow pattern began, which was first demonstrated using ICG lymphography. The lymphatic vessels at the LVA site were then confirmed using ultrasonography. ${ }^{3,11}$ No patients were allergic to iodine.

\section{Real-Time Imaging of the Lymphatic Vessel Using Laser Tomography}

Surgeries were performed under local or general anesthesia and were assisted by EnFocus Ultra-HD optical coherence 
tomography (Leica Microsystems, Wetzlar, Germany) system which was combined with Proveo 8 microscope (Leica Microsystems, Wetzlar, Germany), thus allowing intraoperative real-time laser tomographic imaging with immediate feedback. The black and white scale expresses the strength of the reflection of near-infrared light against subject objects in this system. White color represents the most intense reflection, and black color represents the weakest reflection. This system provides an imaging depth of $2.5 \mathrm{~mm}$ at an optical axial resolution of $<4 \mu \mathrm{m}$. Images are acquired at 32,000 cross-sectional scans per second. It allows continuous visualization in a crosshair aiming mode or acquisition of volumes of up to $1,000 \times 1,000$ lateral pixels. The scan length is up to $16 \mathrm{~mm}$. The total magnification of this microscope is $\times 5.1$ to $\times 30.7$ with $\times 12.5$ eyepiece.

Dynamic scan is controlled via footswitch for swift adjustment of the scan angle to align with the ICG-enhanced lymphatic vessels. Imaging data are displayed on external screen-based display immediately after completion of scanning (- Video $\mathbf{1}$ ). A trained mechanical engineer or nurse assisted intraoperatively with laser tomographic image acquisition and collecting surgeon feedback and data ( - Fig. 1). The imaging findings of the lymphatic vessels in laser tomography were investigated through this procedure using papaverine for preventing contraction of the vessels which leads to thickening of the wall. Using the obtained cross-sectional images of the lymphatic vessels, the diameter of the lumen was measured and recorded during surgery. Also, the thickness of the lymphatic vessel wall was measured at four points and the average was calculated and recorded (-Figs. $\mathbf{2}$ and $\mathbf{3}$ ).

\begin{abstract}
Video 1
Imaging data of the lymphatic vessel was displayed on external screen-based display immediately using laser tomography axial and sagittal scanning. Online content including video sequences viewable at: https://www.thieme-connect.com/products/ ejournals/html/10.1055/s-0040-1718549.
\end{abstract}

After laser tomography scanning, sagittal and axial B-mode ultrasonographic scanning of the lymphatic vessels were performed intraoperatively on the dissected lymphatic vessel using Vevo MD ultrasound device (FUJIFILM VisualSonics, Amsterdam, the Netherlands) with a $70-\mathrm{MHz}$ linear array transducer as previously reported. ${ }^{6}$ The probe of the ultrasound device was placed directly on the lymphatic vessel, which was soaked in saline, and the scan length was up to $15 \mathrm{~mm}$. Required time for scanning of the lymphatic vessels was compared between using laser tomography and ultrasound. The value was reported as mean \pm standard deviation. Differences in the means between groups were analyzed by Mann-Whitney's $U$ test. The $p$-value was two sided, and statistical significance was accepted at $p<0.05$.

\section{Histological Examination}

A small portion of the ICG-enhanced lymphatic vessels which were used for LVA were obtained and sent to histology. The specimens were fixed in 10\% neutral buffered formalin, embedded in paraffin, and sectioned at $4 \mu \mathrm{m}$. The samples

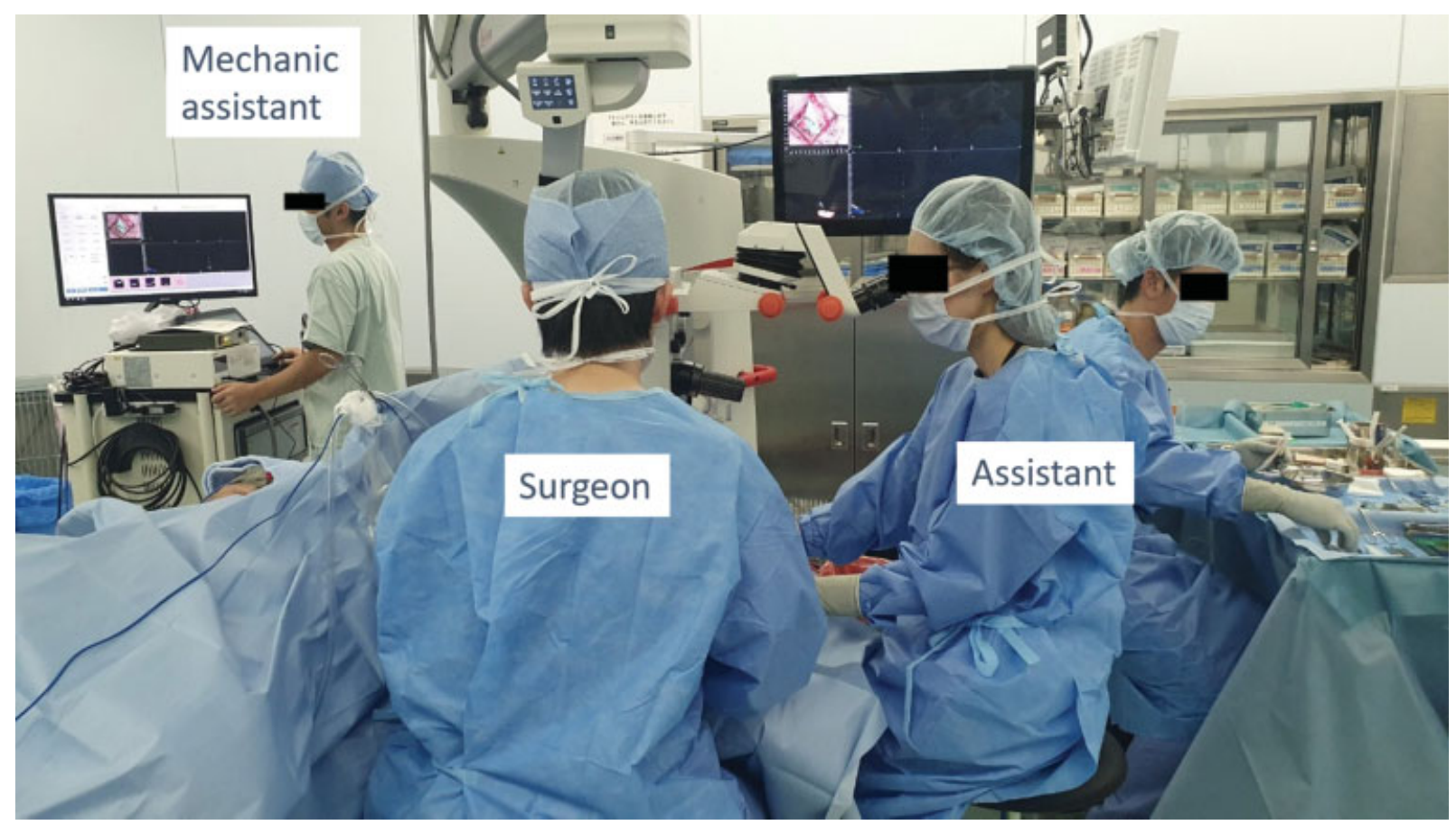

Fig. 1 A trained mechanical engineer assisted intraoperatively with real-time microscope-integrated laser tomography image acquisition. Imaging data are displayed on an external screen-based display immediately after completion of scanning of the lymphatic vessels. 


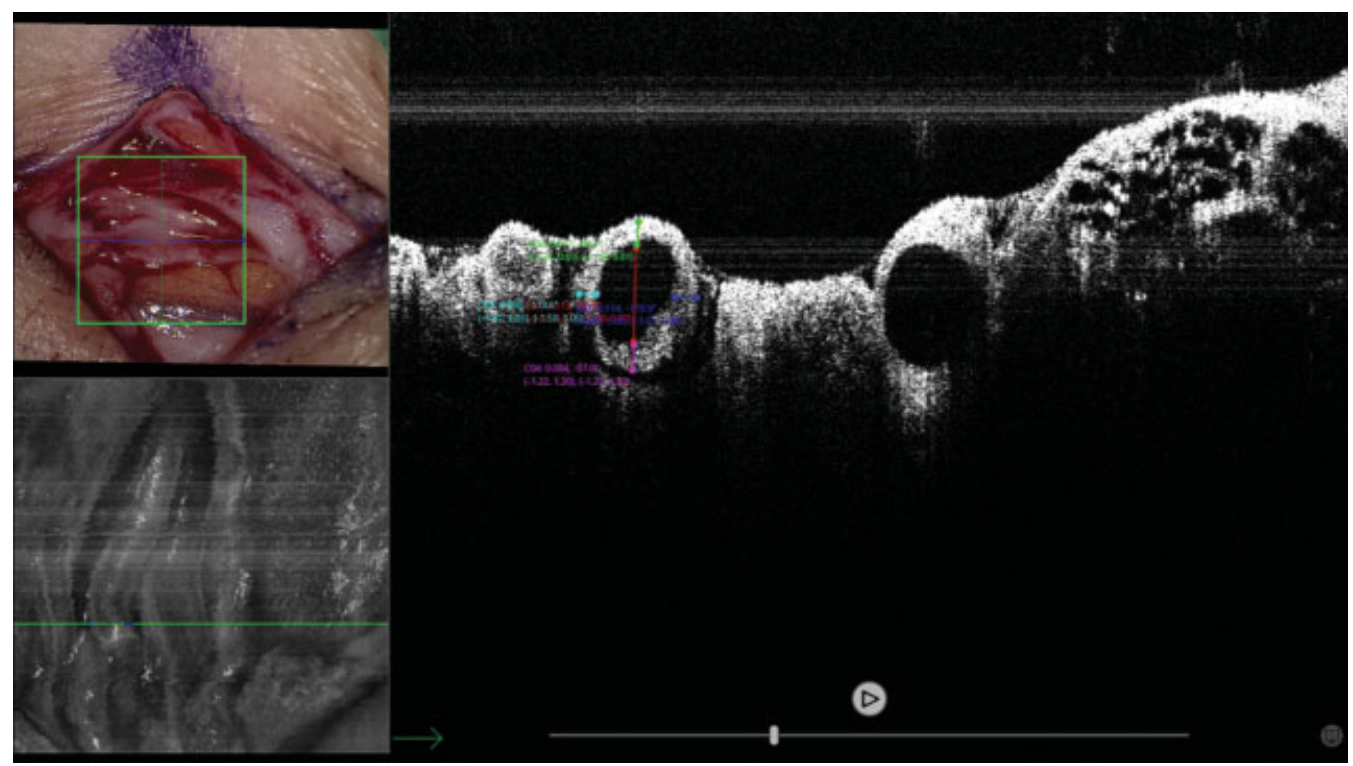

Fig. 2 The diameter of the lumen and the thickness of the lymphatic vessel wall were measured and recorded during surgery using the obtained cross-sectional images of the lymphatic vessels in microscope-integrated laser tomography.
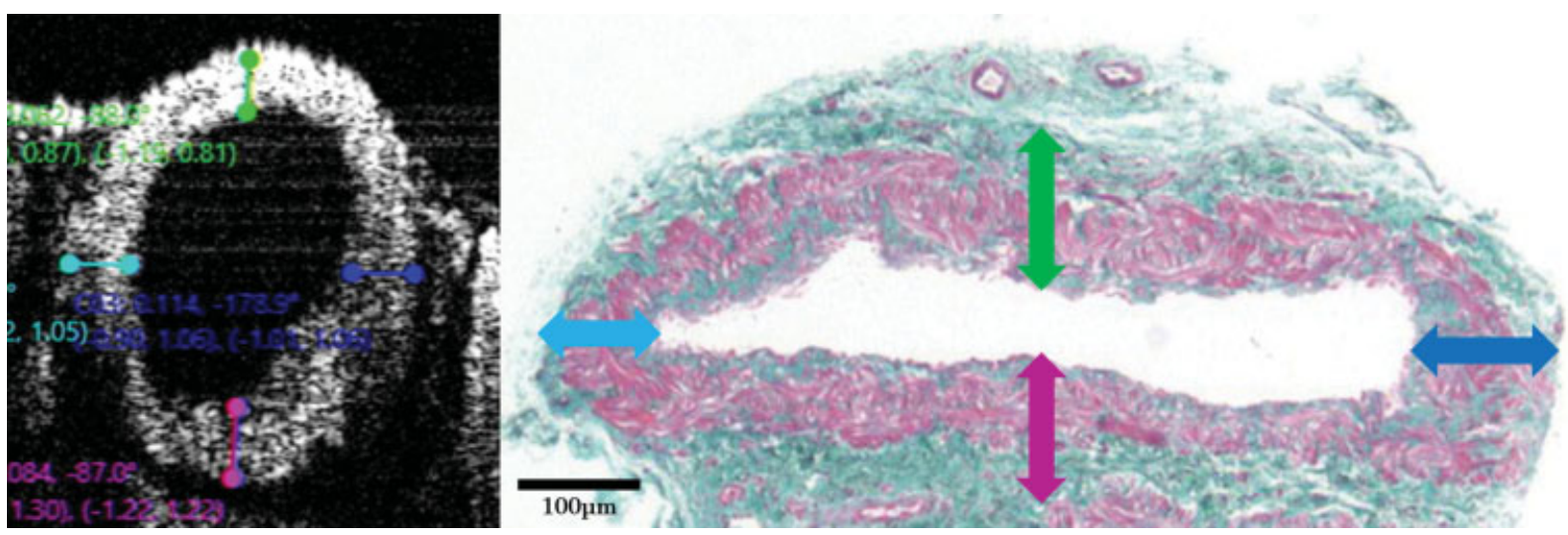

Fig. 3 (Left) The thickness of the lymphatic vessel wall was measured at four points during surgery with microscope-integrated laser tomography. (Right) The thickness of the lymphatic vessels wall, which was histochemical stained with Elastica-Masson stain, was measured at four equally distributed points by histologists.

were stained with hematoxylin-eosin and examined under microscope. In addition, histochemical staining with Elastica-Masson stain was performed for evaluation of degenerative changes of smooth muscle cells and hyperplasia of collagenous fibers in the lymphatic vessels. ${ }^{12}$ The thickness of the lymphatic vessel wall, including collagenous fiber layers and smooth muscle cells, was measured at four equally distributed points by histologists and their average was calculated and documented (-Fig. 3). The correlation between the thickness of the lymphatic vessel wall obtained via laser tomography and the histologically measured thickness was examined. Pearson's correlation coefficient $(r)$ value, 95\% confidence interval $(\mathrm{CI})$, and two-tailed $p$-value were calculated. Good to excellent correlation was defined as an $r$ value $>0.75$, moderate to good as 0.5 to 0.75 , weak as 0.25 to 0.5 , and little or no correlation as $<0.25$. All statistical analyses of recorded data were performed using the Excel statistical software package (Ekuseru-Toukei 2019; Social Survey Research Information Co., Ltd., Tokyo, Japan).

\section{Patency Assessment of Anastomosis Sites Using Laser Tomography}

End-to-end and side-to-end anastomosis sites were scanned using laser tomography immediately after establishment of LVA. Patency was determined based on the images.

\section{Postoperative Assessment of Patency and Volume Changes}

The patency of the anastomosis and arm volume changes were assessed 12 months after the procedure. The patency of the LVA was assessed by observing the scar of the anastomosis sites using the infrared camera system immediately after injection of ICG. Gentle pressure was applied to the lymphatic vessel distal to the anastomosis site to facilitate passage of the lymphatic fluid through the anastomosis site. ${ }^{13}$ The patency status was classified in the following three groups: patent, nonpatent, and not seen. The assessment of the score was done by two independent researchers. The LVA site was regarded as patent only when a clear flush of ICG through the 
anastomosis site was observed. The patency rate was indicated by percentages.

The arm volume changes in terms of circumference were measured according to the upper extremity lymphedema index (UEL index). ${ }^{14}$ After circumference measurement at standardized landmarks on the arm (olecranon, $5 \mathrm{~cm}$ above and below olecranon, wrist, dorsum of the hand), the UEL index was calculated from these circumference points and body mass index (BMI). Patients were asked to remove their compression garments 24 hours prior to the follow-up to achieve a reliable measurement. The variable was obtained preoperatively and 12 months postoperatively. For the arm volume changes, a paired samples $t$-test was used to examine effects pre- and postoperatively.

\section{Results}

Patients' age ranged from 33 to 81 years (median, 53 years), BMI ranged from 17.7 to $34.2 \mathrm{~kg} / \mathrm{m}^{2}$ (median, $25.6 \mathrm{~kg} / \mathrm{m}^{2}$ ), and duration of edema ranged from 4 to 252 months (median, 21 months). Twenty-three patients (44.2\%) had a history of radiation therapy, and 20 patients (38.5\%) had a history of cellulitis. International Society of Lymphology stage was determined as stage I in 12 cases (23.1\%), stage Ila in 19 cases (36.5\%), stage IIb in 17 cases (32.7\%), and stage III in 4 cases (7.7\%). Dermal backflow stage was determined as stage 0 in 1 case (1.9\%), stage I in 6 cases (11.5\%), stage II in 11 cases (21.2\%), stage III in 18 cases (34.6\%), stage IV in 11 cases (21.2\%), and stage $\mathrm{V}$ in 5 cases (9.6\%).

A total of 132 ICG-enhanced lymphatic vessels were found in 117 incisions: 28 in the upper arm, 97 in the forearm, and 7 in the hand. These lymphatic vessels were scanned with laser tomography before LVA. The lymphatic vessel wall was demonstrated as white region and the lumen of the lymphatic vessel was demonstrated as black region in laser tomography images. The thickness of the lymphatic vessel wall ranged from 0.021 to $0.168 \mathrm{~mm}$ (median, $0.092 \mathrm{~mm}$ ), and the diameter of lymphatic vessels ranged from 0.034 to $0.487 \mathrm{~mm}$ (median, $0.196 \mathrm{~mm}$ ). In 59 lymphatic vessels (44.7\%), valves, which were seen as white winding lines, were found inside the lumen (-Fig. 4 ).

Significant difference was seen between the required time for scanning of the lymphatic vessels using laser tomography and ultra-high-frequency ultrasound (1.6 \pm 0.3 vs. $4.8 \pm 1.2$ minutes; $p=0.016$ ).

The correlation between the thickness of lymphatic vessel wall measured with laser tomography and histologically measured thickness was good to excellent $(r=0.977,95 \%$ CI: 0.897-0.992, $p<0.001$ ) (-Fig. 5).

Patency of the anastomosis sites could be determined in all anastomoses using laser tomography (107 end-to-end anastomoses and 25 side-to-end anastomoses) (-Figs. 6 and 7; - Video 2). Four anastomosis sites demonstrated inverted edges of the vessels, and thus were immediately reanastomosed (-Fig. 8).

\section{Video 2}

Patency of the anastomosis sites was determined in side-to-end anastomosis between lymphatic vessel and vein using laser tomography scanning. Online content including video sequences viewable at: https://www.thieme-connect.com/products/ ejournals/html/10.1055/s-0040-1718549.

Postoperative examination with ICG lymphography demonstrated patent in 110 out of 132 anastomoses (83.3\%), nonpatent in 4 anastomoses (3.6\%), and not seen in 18 anastomoses (13.6\%). The volume difference in terms of

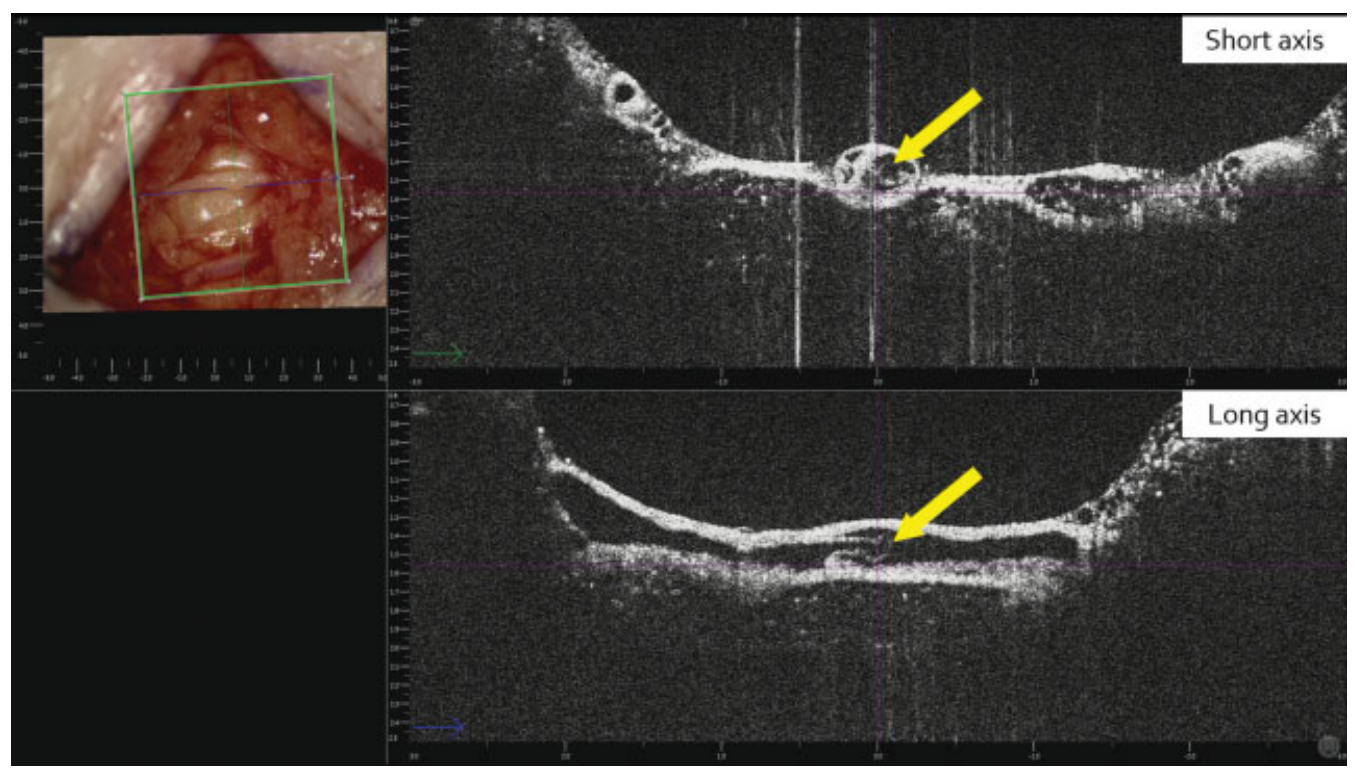

Fig. 4 Valves (yellow arrows), which were seen as white winding lines, were found inside the lumen of the lymphatic vessels with the obtained cross-sectional images of the lymphatic vessels in microscope-integrated laser tomography. 


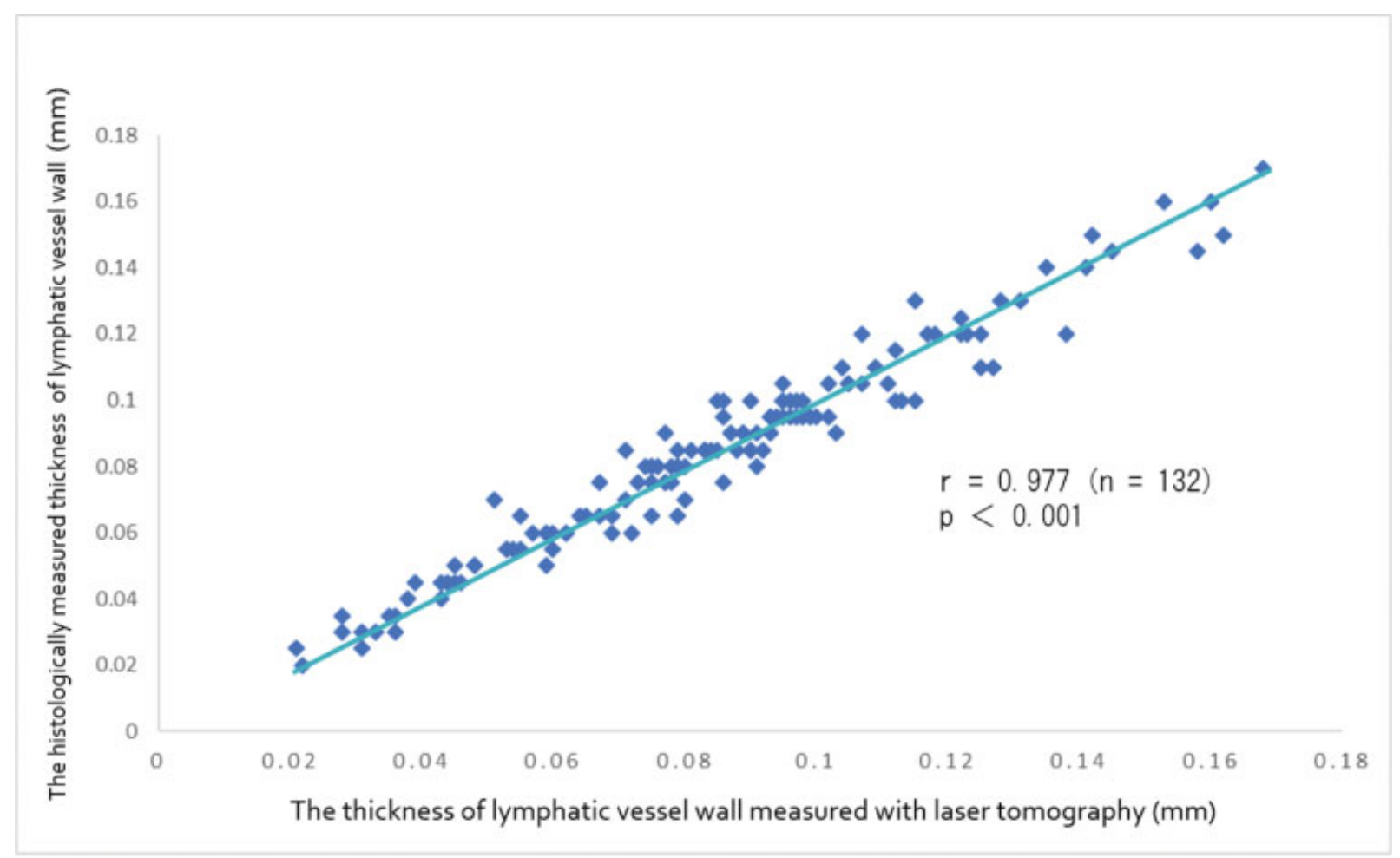

Fig. 5 The correlation between the thickness of lymphatic vessel wall measured with laser tomography and histologically measured thickness.

UEL index between the affected and unaffected arms was 14.3 preoperatively and 10.8 postoperatively, respectively. The difference reached statistical significance $(p<0.05)$.

\section{Discussion}

The present study was conducted to reveal the new capabilities of laser tomography for intraoperative imaging of the lymphatic vessels during LVA. From the results of this study, laser tomography not only showed unprecedented clear images of the lymphatic vessels in all operative sites, even with the vessels with diameters smaller than $0.3 \mathrm{~mm}$, but also demonstrated high ability for intraoperative evaluation of lymphatic degeneration status, putting us a step closer in choosing the ideal lymphatic vessel for LVA. This obviously confers the ability to select the best lymphatic vessel on surgeons.

To date, ICG fluorescence imaging modalities are often used for intraoperative detection and selection of lymphatic vessels. ${ }^{5}$ However, ICG is not available in all institutions and is contraindicated for patients with allergy to iodine. Yang et al reported that lymphatic flow-positive but non-ICG-enhanced lymphatic vessels should also be considered as functional lymphatic vessels. In fact, some non-ICG-enhanced lymphatic

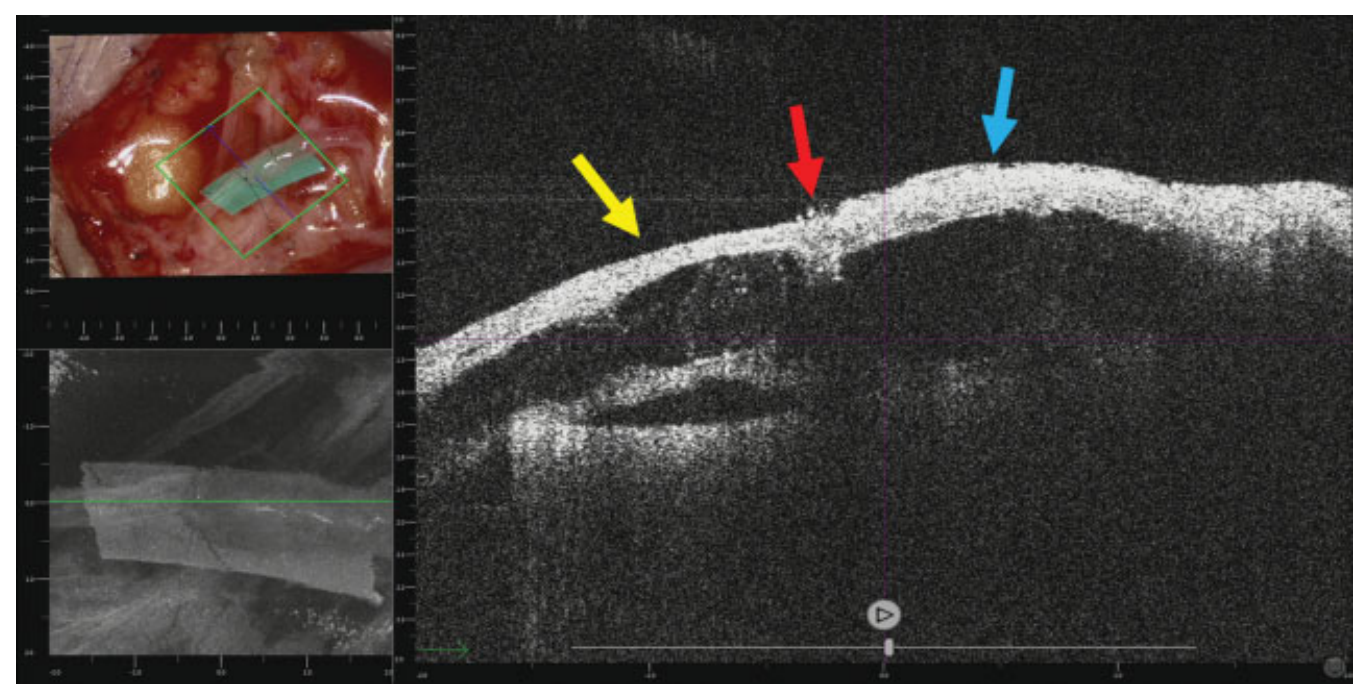

Fig. 6 Patency of the anastomosis sites (red arrow) was determined in end-to-end anastomosis between lymphatic vessel (yellow arrow) and vein (blue arrow) using sagittal scanning of laser tomography. 


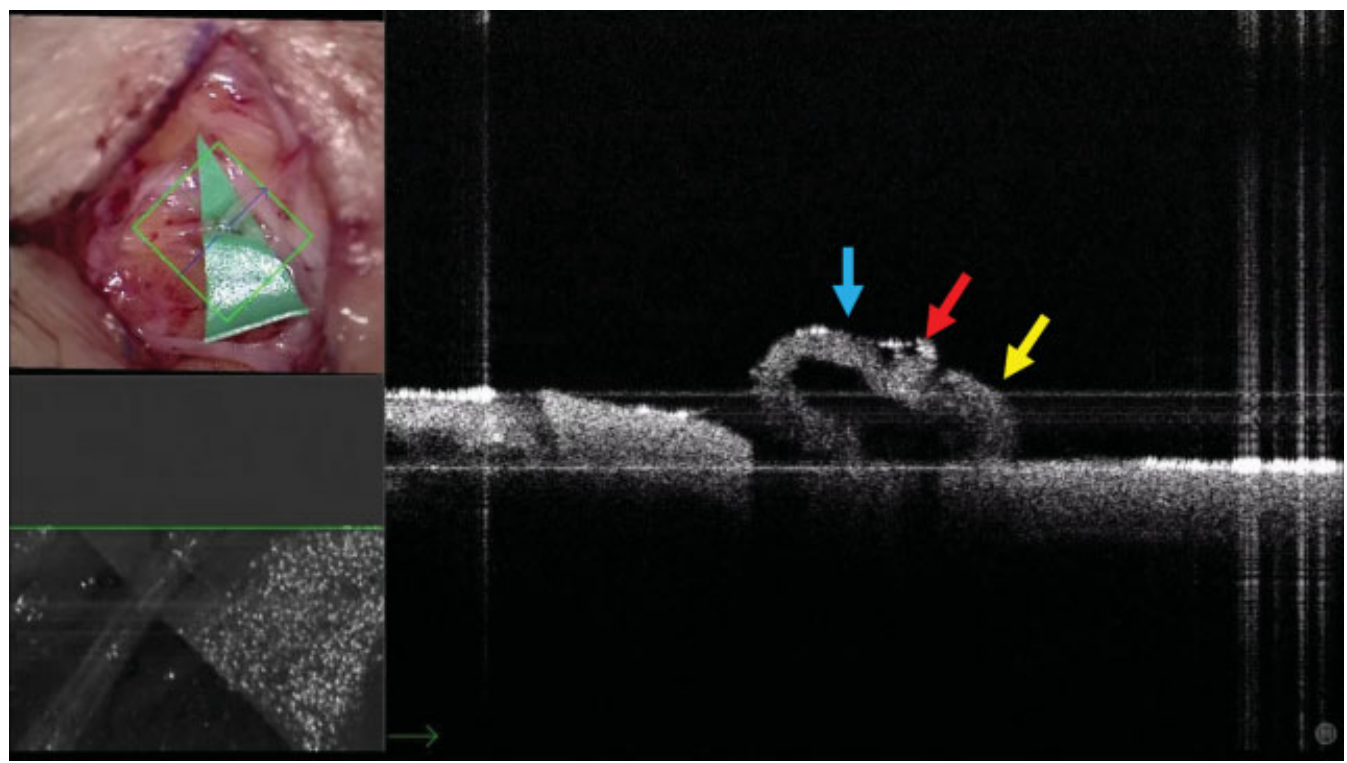

Fig. 7 Patency of the anastomosis sites (red arrow) was determined in side-to-end anastomosis between lymphatic vessel (yellow arrow) and vein (blue arrow) using axial scanning of laser tomography.

vessels were detected using laser tomography in this study. From these reasons, a new modality for finding the functional lymphatic vessels was warranted. Although Hayashi et al reported noninvasive intraoperative imaging of the lymphatic vessels using ultra-high-frequency ultrasonography, this technique is time consuming and highly operator dependent.. In this study, microscope-integrated laser tomography was less time consuming for intraoperative imaging of the lymphatic vessels than ultrasonography because all procedures, including simultaneous sagittal and axial scanning of the lymphatic vessels, were automated.

After breast cancer treatments, lymph flows are obstructed in the axilla, and lymphatic hypertension takes place. ${ }^{10,15}$ Lymphatic hypertension causes dilatation of the lymphatic vessels, lymphatic valvular insufficiency, retrograde lymph

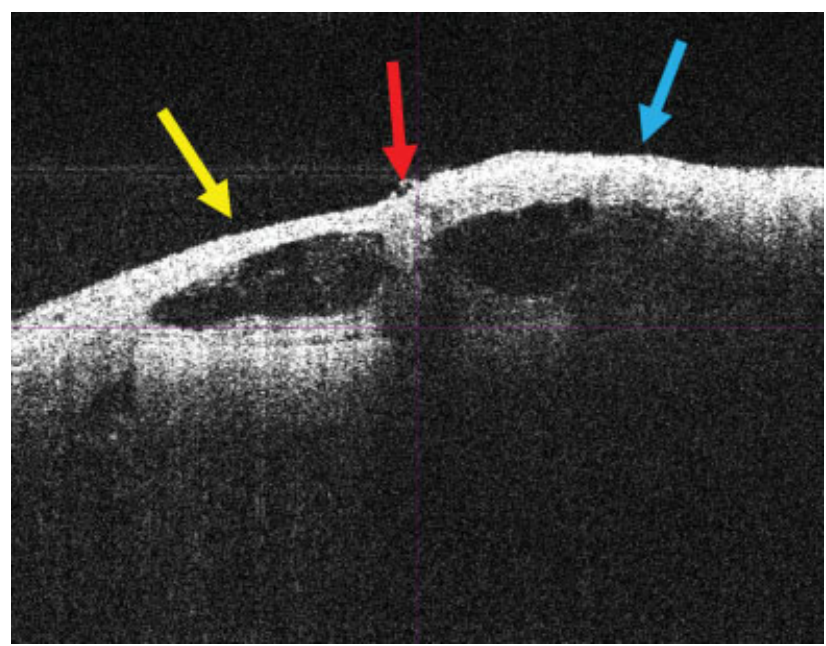

Fig. 8 Patency of the end-to-end anastomosis site (red arrow) between the lymphatic vessel (yellow arrow) and the vein (blue arrow) using sagittal scanning of laser tomography demonstrated inverted edges of the vessels. flows, and inflicts further mechanical stresses on the lymphatic vessels. ${ }^{16,17}$ The lymphatic vessels become sclerotic over time after lymph flow obstruction, and lose their function to drain lymph fluid. ${ }^{16,18}$ It is essential to select the best functional lymphatic vessels with less sclerosis in LVA for maximum therapeutic effects. ${ }^{3-5}$ According to previous immunohistochemical and electron microscopic studies, lymphosclerosis is characterized by discontinuity of the endothelium, degenerative changes of smooth muscle cells, and hyperplasia of collagenous fibers. ${ }^{19,20}$ Some previous studies reported that classification of the lymphatic vessel condition is possible by intraoperative observation of the transected lymphatic vessels under microscope. ${ }^{2,17}$ However, assessment of intraoperative findings under microscope can be subjective, requiring surgical experience. This study revealed that laser tomography can provide objective information regarding the thickness of lymphatic vessel wall, which correlated with histologically measured thickness. In addition, laser tomography could clearly visualize and identify the valves of the lymphatic vessels in some cases. Using laser tomography, even surgeons with less experience can choose the most suitable lymphatic vessels, including those not highlighted with ICG, in the surgical field just by scanning the region (-Fig. 9).

As of now, in all previous reports, patency of the anastomosis sites was evaluated via intraoperative ICG observation, where, in most cases, the lymphatic vessel distal to the anastomosis site was "milked" to push the lymphatic fluid through the anastomosis site. While this procedure may be optimal for assessment of leakage in the anastomosis site, the patency will be determined as positive even in anastomoses with a minimum patent lumen. Furthermore, there are some functioning lymphatic vessels in which ICG is not taken up, making evaluation difficult. Due to extremely high resolution of the images that can be obtained with laser tomography, the patency of the anastomosis sites can be 


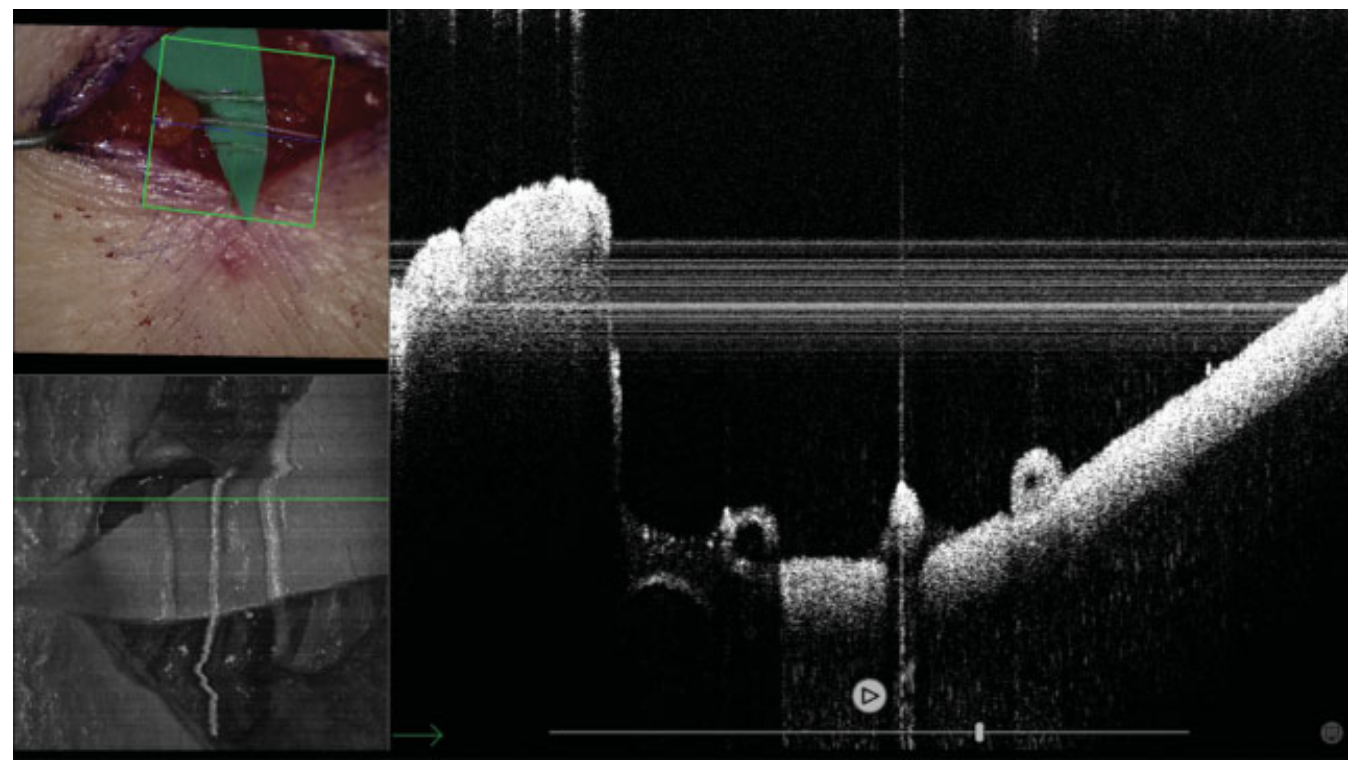

Fig. 9 Although three lymphatic vessels were similar in appearance under microscope, microscope-integrated laser tomography showed that they have different lymphatic degeneration status.

more precisely determined, thus possibly leading to higher efficacy of LVA. In this study, some anastomosis sites demonstrated inverted edges of the vessels, which necessitated immediate reanastomosis even though the patency was confirmed with ICG observation. Laser tomography may also be useful for beginner of lymphatic surgery because it provides immediate feedback of the anastomosis status.

A disadvantage of this new imaging modality is its limited reaching distance of near-infrared light. The deepest layer from which the device can obtain images is $2.5 \mathrm{~mm}$ from the superficial surface after incision. This precludes its use in preoperative examination, but the reaching distance is sufficient for intraoperative use, where the lymphatic vessels are already dissected out.

Limitations of this study include that the study was a retrospective observational study that included only female patients with upper extremity lymphedema secondary to breast cancer treatments who underwent LVA surgery. It is unclear whether similar results can be obtained in studies including lower extremity peripheral lymphedema, male patients, or primary lymphedema. It is also yet to be clarified whether the use of laser tomography improves the therapeutic efficacy of LVA. In this study, identification rates of the laser tomography were not compared with those of ICG lymphography because it was a preliminary investigational study for confirming feasibility of laser tomography. Further studies are required to address these questions.

\section{Conclusion}

To the best of our knowledge, this is the first attempt to detect the lymphatic vessels with laser tomography intraoperatively during LVA. This novel device provides real-time images of the lymphatic vessels in extremely high resolution and enables intraoperative evaluation of lymphatic degeneration status. In addition, patency of the anastomosis sites can be determined with extreme precision. This advanced technology for lymphedema may open new frontiers in understanding and treatment of this complex disease.

\section{Authors' Contributions}

Conception and design: A.H., H.Y., and J.P.H.; analysis and interpretation: A.H., H.Y., G.V., G.G., N.H., T.Y., and J.C.Y.; data collection: A.H., H.Y., and S.S.; statistical analysis: A. H., H.Y., G.V., and S.S.; writing the manuscript: A.H., H.Y., and J.P.H.; critical revision of the manuscript: A.H., H.Y., G. V., J.C.Y., and J.P.H.; final approval of the manuscript: A.H., H.Y., G.V., G.G., T.Y., J.C.Y., and J.P.H.

\section{Note}

This study was presented at the 2020 American Society of Reconstructive Microsurgery Annual Meeting.

\section{Conflict of Interest}

None declared.

\section{References}

1 Koshima I, Inagawa K, Urushibara K, Moriguchi T. Supermicrosurgical lymphaticovenular anastomosis for the treatment of lymphedema in the upper extremities. J Reconstr Microsurg 2000;16 (06):437-442

2 Salgarello M, Mangialardi ML, Pino V, Gentileschi S, Visconti G. A prospective evaluation of health-related quality of life following lymphaticovenular anastomosis for upper and lower extremities lymphedema. J Reconstr Microsurg 2018;34(09):701-707

3 Hayashi A, Hayashi N, Yoshimatsu H, Yamamoto T. Effective and efficient lymphaticovenular anastomosis using preoperative ultrasound detection technique of lymphatic vessels in lower extremity lymphedema. J Surg Oncol 2018;117(02):290-298

4 Czedik-Eysenberg M, Steinbacher J, Obermayer B, et al. Exclusive use of ultrasound for locating optimal LVA sites-a descriptive data analysis. J Surg Oncol 2020;121(01):51-56

5 Yang JC, Wu SC, Chiang MH, et al. Intraoperative identification and definition of "functional" lymphatic collecting vessels for 
supermicrosurgical lymphatico-venous anastomosis in treating lymphedema patients. J Surg Oncol 2018;117:994-1000

6 Cho MJ, Kwon JG, Pak CJ, Suh HP, Hong JP. The role of duplex ultrasound in microsurgical reconstruction: re. view and technical considerations. J Reconstr Microsurg 2020;36(07):514-521

7 Hayashi A, Visconti G, Yamamoto T, et al. Intraoperative imaging of lymphatic vessel using ultra high-frequency ultrasound. J Plast Reconstr Aesthet Surg 2018;71(05):778-780

8 Chen TC, Cense B, Pierce MC, et al. Spectral domain optical coherence tomography: ultra-high speed, ultra-high resolution ophthalmic imaging. Arch Ophthalmol 2005;123(12):1715-1720

9 Hee MR, Izatt JA, Swanson EA, et al. Optical coherence tomography of the human retina. Arch Ophthalmol 1995;113(03):325-332

10 Heydon-White A, Suami H, Boyages J, Koelmeyer L, Peebles KC. Assessing breast lymphoedema following breast cancer treatment using indocyanine green lymphography. Breast Cancer Res Treat 2020;181(03):635-644

11 Burnier P, Niddam J, Bosc R, Hersant B, Meningaud JP. Indocyanine green applications in plastic surgery: a review of the literature. J Plast Reconstr Aesthet Surg 2017;70(06):814-827

12 O'Connor WN, Valle S. A combination Verhoeff's elastic and Masson's trichrome stain for routine histology. Stain Technol 1982;57(04):207-210

13 Wolfs JAGN, de Joode LGEH, van der Hulst RRWJ, Qiu SS. Correlation between patency and clinical improvement after lymphaticovenous anastomosis (LVA) in breast cancer-related lymphedema: 12-month follow-up. Breast Cancer Res Treat 2020;179(01):131-138
14 Yamamoto N, Yamamoto T, Hayashi N, Hayashi A, Iida T, Koshima I. Arm volumetry versus upper extremity lymphedema index: validity of upper extremity lymphedema index for body-type corrected arm volume evaluation. Ann Plast Surg 2016;76(06): 697-699

15 Yamamoto T, Yamamoto N, Doi K, et al. Indocyanine greenenhanced lymphography for upper extremity lymphedema: a novel severity staging system using dermal backflow patterns. Plast Reconstr Surg 2011;128(04):941-947

16 Koshima I, Kawada S, Moriguchi T, Kajiwara Y. Ultrastructural observations of lymphatic vessels in lymphedema in human extremities. Plast Reconstr Surg 1996;97(02):397-405, discussion 406-407

17 Yamamoto T, Yamamoto N, Yoshimatsu H, Narushima M, Koshima I. LEC score: a judgment tool for indication of indocyanine green lymphography. Ann Plast Surg 2013;70(02):227-230

18 Yamamoto $\mathrm{T}$, Narushima $\mathrm{M}$, Yoshimatsu $\mathrm{H}$, et al. Indocyanine green velocity: lymph transportation capacity deterioration with progression of lymphedema. Ann Plast Surg 2013;71(05): 591-594

19 Mihara M, Hara H, Hayashi Y, et al. Pathological steps of cancerrelated lymphedema: histological changes in the collecting lymphatic vessels after lymphadenectomy. PLoS One 2012;7(07): e41126

20 Boccardo F, Fulcheri E, Villa G, et al. Lymphatic microsurgery to treat lymphedema: techniques and indications for better results. Ann Plast Surg 2013;71(02):191-195 